\section{Pacific Northwest}

National Laboratory

Operated by Battelle for the

U.S. Department of Energy

\title{
Sorptive Polymers and Photopatterned Films for Gas Phase Chemical Microsensors and Arrays
}

J. W. Grate*
D. A. Nelson*

Environmental Molecular Sciences Laboratory Richland, Washington 99352

October 2002

Prepared for the U.S. Department of Energy under Contract DE-AC06-76RL01830 


\title{
DISCLAIMER
}

This report was prepared as an account of work sponsored by an agency of the United States Government. Neither the United States Government nor any agency thereof, nor Battelle Memorial Institute, nor any of their employees, makes any warranty, express or implied, or assumes any legal liability or responsibility for the accuracy, completeness, or usefulness of any information, apparatus, product, or process disclosed, or represents that its use would not infringe privately owned rights. Reference herein to any specific commercial product, process, or service by trade name, trademark, manufacturer, or otherwise does not necessarily constitute or imply its endorsement, recommendation, or favoring by the United States Government or any agency thereof, or Battelle Memorial Institute. The views and opinions of authors expressed herein do not necessarily state or reflect those of the United States Government or any agency thereof.

\author{
PACIFIC NORTHWEST NATIONAL LABORATORY \\ operated by \\ BATTELLE \\ for the \\ UNITED STATES DEPARTMENT OF ENERGY \\ under Contract DE-AC06-76RL01830
}

This document was printed on recycled paper. 
PNNL-14124

\title{
Sorptive Polymers and Photopatterned Films for Gas Phase Chemical Microsensors and Arrays
}

\author{
J. W. Grate* \\ D. A. Nelson* \\ * Environmental Molecular Sciences Laboratory \\ Richland, WA 99352
}

October 2002

Prepared for the U.S. Department of Energy under Contract DE-AC06-76RL01830

Pacific Northwest National Laboratory

Richland, Washington 99352 


\section{Summary}

The selective layer on a gas phase chemical microsensor plays a critical role in the sensitivity and selectivity of the sensor's response. Polymers can rapidly and reversibly absorb vapor molecules. As sorptive layers on chemical sensors, polymers are useful for a variety of chemical microsensor types and transduction mechanisms, either as is or as composites with conductive particles or dyes. The performance requirements for polymers are described in terms of their chemical and physical properties. A versatile synthetic approach for preparing sensing polymers has been developed using hydrosilylation chemistry to prepare carbosiloxane polymers. Polymers with diverse chemical selectivities have been prepared by this method. Furthermore, sorptive polymer materials can be photopatterned into defined areas on surfaces using a photoactivated catalyst and hydrosilylation chemistry. Polymer films with diverse chemical selectivities have been patterned with this new method. 


\section{Acknowledgements}

The authors thank Glen Dunham for photopatterning the polydimethylsiloxane formulations and for assistance in the clean room. The authors are grateful for support from the U.S. Department of Energy Office of Nonproliferation Research and Engineering, NA-22. The Pacific Northwest National Laboratory is a multiprogram national laboratory operated for the U. S. Department of Energy by Battelle under Contract DE-AC06-76RL01830. 


\section{Contents}

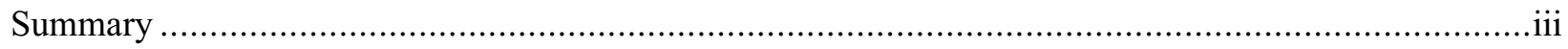

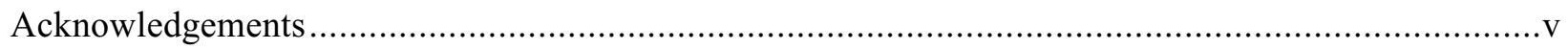

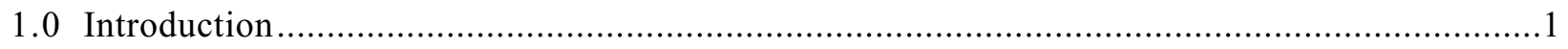

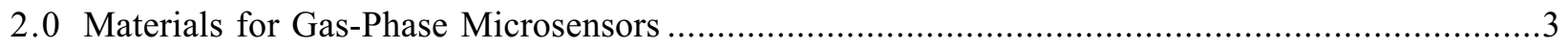

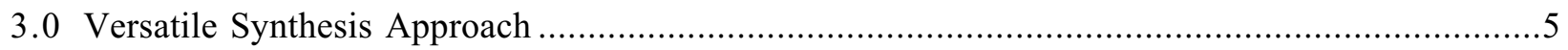

4.0 Photopatterning Sorptive Polymeric Layers ............................................................13

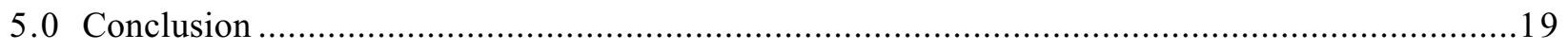

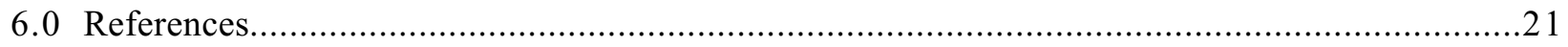

Appendix A: Detailed Experimental Procedures.................................................................. A.1

\section{Figures}

1. Schematic Diagram of a Typical Coated Microsensor Where the Selective Layer Collects and Concentrates Vapor Molecules at the Sensor Surface by Reversible Sorption into the Layer Material

2. Schematic Diagram of a Sensor Array, Where each Sensor is Coated with a Different

Selective Layer.

3. Hydrosilylation Chemistry, Where a) Shows the Hydrosilylation Reaction Adding Si-H Bond Across a C-C Double Bond, and b) Shows Hydrosilylation Polymerization Involving a Monomer with two C-C Double Bonds and a Second Monomer that is an $\alpha, \omega-$ dihydridooligosiloxane with Si-H Bonds on each End.

4. The Chemical Structures of some Monomers Used for Making Carbosiloxane Polymers........ 7

5. Selected Examples of Carbosiloxane Polymers Prepared for Selective Layers on Vapor Sensors, Illustrating a Wide Range of Chemical Structures and Interactive Properties ..... .8

6. Comparison of the Response Time Behavior of Two Polymers, BSP3 and FPOL, to Toluene. Data points are two seconds apart......

7. Calibration Curves Comparing the Sensitivities of Three Polymers (BSP3, FPOL, and PDMS) to dimethyl methylphosphonate (DMMP). 
8. Schematic Diagram of the Photopatterning Process Where Those Domains Exposed to UV Light are Crosslinked and Retained on the Surface After Washing with a Developing Solvent, Whereas Those Domains Not Exposed Are Washed Away.....

9. Repeat Unit Structures of some Vinyl-Containing Polyorganosiloxane Polymers Used for Making Photopatterned Films

10. Structures of Some Crosslinkers Used for Making Photopatterned Films

11. Structure of the Si-H Terminated BSP3 Oligomer Used for Making Photopatterned BSP-Containing Films 16

12. Images of Photopatterned Polymer Films 17

\section{Table}

1. Formulations for Photopatterned Sorbent Films ${ }^{(a)}$ 16 


\subsection{Introduction}

Chemical sensors using interactive sorptive materials typically involve a "two-step" response mechanism. While the sorptive material collects and concentrates gas phase molecules, the device on which it has been applied transduces the presence of these sorbed molecules into an analytical signal. Usually, the sorptive material is a thin film and the device is a microfabricated structure, although other configurations exist. The film of a sorptive material is often referred to as the "selective layer". This general concept for gas phase chemical sensing is shown in Figure 1.

A variety of devices and structures can be used for signal transduction, including acoustic wave, chemiresistor, and optical approaches. Regardless of the transduction mechanism, the sorptive material plays a critical role in determining the sensitivity and selectivity of the sensor. Acoustic wave devices transduce the mass of the sorbed vapor, and sometimes its effect on polymer viscoelastic properties, into an analytical signal; thus their selectivity is largely due to the selectivity with which vapors are sorbed by the sorptive coating material. Chemiresistors require a layer that is electronically conductive, and responds to species that are sorbed by and alter the conductivity of the selective layer. Optical chemical sensors can respond to a variety of selective layer changes, including refractive index, optical absorbance, and fluorescence. In the acoustic wave sensor field, there have been several reviews on mechanisms for chemical selectivity and chemically selective layers for various gas phase detection applications.[110]

Many of these sensor types are used in sensor arrays.[11-13] Arrays offer greater selectivity than single sensors, since the response patterns of an array of semiselective sensors contain much more chemical information than the responses of any single sensor. When arrays are used, several different sensing materials, each with somewhat different selectivity, are required. The concept of a sensor array is shown in Figure 2. The set of selective layers in the array should maximize the collection of relevant chemical information for detecting and discriminating analytes of interest. 
The sorptive materials highlighted in this article will be primarily polymeric materials, with emphasis on acoustic wave sensor applications. Acoustic wave devices include surface acoustic wave, or SAW, devices, as well as others such as thickness shear mode (TSM) devices and flexural plate wave (FPW) devices. Acoustic wave sensors provide a very sensitive means of transducing the sorption of vapors by nonconductive polymeric materials. Mechanisms of response for acoustic wave sensors have been described in a number of articles, reviews, and books.[14-18] Use of acoustic wave sensors in arrays has recently been reviewed.[12]

However, sorptive polymeric materials are equally useful for chemiresistor sensors if formulated as composites with conducting particles; then the composite material is electronically conductive and vapor sorption is transduced as a change in resistance across the sensor. This approach to chemiresistor sensing using polymers was reported in the 1980 's[19, 20] and has more recently been used for the development of sensor arrays.[11, 21-24]

Polymeric materials have also been widely used as selective layers for optical sensors. Refractive index changes in the polymer as a function of sorbed vapor can be transduced using a variety of configurations including planar optical waveguides and evanescent wave optical fiber methods. In addition, polymers can be loaded with dyes for either absorbance or fluorescence measurements. Arrays of optical sensors have been prepared by loading polymer/dye composites on beads and locating these beads on the end of fiber optic bundles.[11, 25-28]

Thus, it is apparent that polymers with good physical and chemical properties for rapid and reversible vapor sorption can be quite useful for vapor sensing, either as insulating materials for acoustic or optical sensing, or as composites for chemiresistor or optical sensing. 


\subsection{Materials for Gas-Phase Microsensors}

A great variety of materials have been employed as layers on the surfaces of chemical microsensors to modify the sensitivity and selectivity for gas phase chemicals. Materials applied range from conventional chromatographic stationary phases and polymers to such unusual materials as soot extracts.[29] Materials used as selective layers on acoustic wave sensors arrays were recently reviewed;[12] these materials included amorphous polymers, dendrimers, self-assembled monolayers, lipids, and cavitands. Whatever the layer material, its functionality depends on the extent to which it enhances the sensitivity and selectivity of the sensor, while still affording reversibility and reproducibility. In sensor array applications, it is particularly important that sensor materials respond rapidly, reversibly, and reproducibly so that response patterns are consistent over time.

Polymers consistently meet these performance requirements and are particularly versatile materials for chemical vapor sensing. They can be applied as thin films and they effectively collect and concentrate vapor molecules on sensor surfaces by reversible sorption. Moreover, their chemical selectivity is determined by chemical structure, which can be easily varied through synthesis. Thus, polymers can be tailored for particular applications, and diverse sets of polymers can be assembled for use in sensor arrays.

Both the chemical and the physical properties of a sensing polymer are important in determining its performance. While the chemical properties influence the selectivity, the physical properties influence other aspects of the performance such as response time. Rapid responses, which are usually desirable for chemical sensors, are promoted by polymers with glass-to-rubber transition temperatures below the operating temperature of the sensor. Vapor diffusion in and out of polymers is rapid when this condition is met, whereas the response times of sensors with glassy polymers are typically much slower than those based on rubbery polymers. In experiments with thin films on SAW devices, slow diffusion in glassy polystyrene has been contrasted with rapid diffusion in and through polysiloxane films,[30] which have very low glass transition temperatures.

Many polymers with low glass transition temperatures are commercially available and can be empirically tested as sensing layers. Gas chromatographic stationary phases such as those based on organosiloxane polymers are useful, as well as commercial materials such as poly(isobutylene) rubber, just to mention a few. Because siloxane linkages in polymers have very high mobility if not excessively hindered, providing polymers with low glass transition temperatures, several authors have noted and used substituted polysiloxanes as sensor phases.[4, 31-33] Arrays can be empirically constructed using sets of commercially-available polymers, and in many applications these arrays will be very effective. Polymers continue to serve as the basis for chemical sensors, sensor arrays, and "electronic nose" approaches, either commercialized or under development.

On the other hand, chemical selectivity can be rationally understood in terms of fundamental interactions that influence vapor sorption, such as dispersion interactions, dipole-induced dipole interactions, dipole-dipole interactions, and hydrogen bonding interactions. Vapor sorption as a function of these interactions has been systematically studied and modeled using linear solvation energy relationships (LSERs).[4, 12, 16, 34] Polymer structures can be rationally designed to participate in particular interactions in order to obtain particular selectivities. In addition, sets of polymers for arrays can be selected so that the array probes all the fundamental interactions that govern vapor absorption. 


\subsection{Versatile Synthesis Approach}

Despite the commercial availability of a variety of sorptive polymers, there remain compelling reasons to synthesize polymers for chemical sensors. These include:

- To prepare materials with selectivities that are not available in commercial polymers

- To put rational design principles to work in sensor material development

- To overcome shortcomings in the performance of commercial polymers as layers on sensors

- To obtain rigorous control over composition and properties, combining the desired chemically interactive properties with necessary physical properties for performance as thin films on sensors

- To prepare polymers and prepolymers for specific thin film processing approaches, such as crosslinking, grafting to surfaces, and photopatterning

- To prepare and adapt polymer and prepolymer formulations for application as sensing thin films on various sensing platforms

Nevertheless, the development of new sensing polymers, one-at-a-time for particular applications, could be costly and time consuming. It was for this reason that we set out to develop a versatile approach for designing and synthesizing sorptive polymers that would afford predictable chemical selectivity and predictable physical properties, while allowing a diversity of materials to be readily prepared.[35] The approach we used is based on using hydrosilylation chemistry as the bond forming reaction during polymerization.[36] The polymers obtained are hybrid organic/inorganic class of polymers called carbosiloxanes.

The hydrosilylation reaction adds $\mathrm{Si}-\mathrm{H}$ bonds across $\mathrm{C}-\mathrm{C}$ double bonds in the presence of a noble metal catalyst such as $\mathrm{Pt}$. The hydrosilylation reaction and its use for making carbosiloxane polymers are shown in Figure 3. The carbosiloxane polymers are derived from the reaction of $\alpha, \omega$ dihydridooligosiloxanes with additional monomers containing two terminal $\mathrm{C}-\mathrm{C}$ double bonds. The hydrosilylation reaction is selective for reaction with $\mathrm{C}-\mathrm{C}$ bonds in the presence of many other functional groups.[37, 38] In addition, the use of oligosiloxane segments in polymers, often leads to low glass-to-rubber transition temperatures, as noted previously for polysiloxanes. Therefore, this polymerization approach meets two important criteria with regard to the chemical and physical properties of sensing polymers: diverse polymers with various functional groups can be prepared, and the resulting polymers should exhibit low glass-to-rubber transition temperatures. Another very desirable feature of this approach is that the bond forming reaction does not bias the selectivity of the resulting material, since it produces only silicon-carbon bonds and does not introduce any polar functionalities into the final material. 

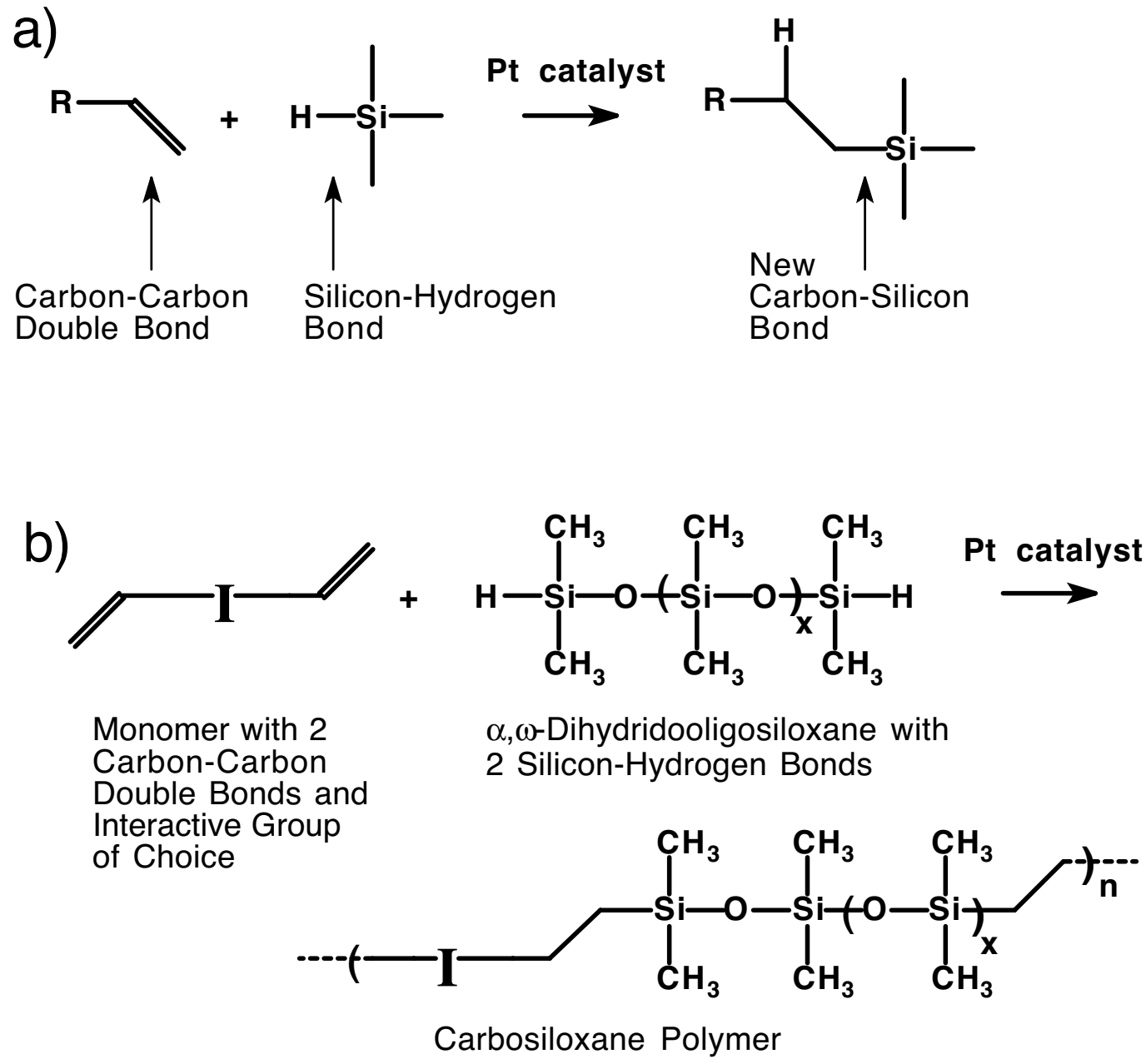

Figure 3. Hydrosilylation Chemistry, Where a) Shows the Hydrosilylation Reaction Adding Si-H Bond Across a C-C Double Bond, and b) Shows Hydrosilylation Polymerization Involving a Monomer with two C-C Double Bonds and a Second Monomer that is an $\alpha, \omega$-dihydridooligosiloxane with $\mathrm{Si}-\mathrm{H}$ Bonds on each End. The monomer with two $\mathrm{C}-\mathrm{C}$ bonds has the interactive (I) properties of choice for chemical selectivity as sensing material.

A variety of monomers can be applied in this synthetic approach, some of which are shown in Figure 4. Many organic compounds with two terminal C-C double bonds exist (or can be designed and synthesized) that could be combined with $\alpha, \omega$-dihydridooligosiloxanes to yield carbosiloxane polymers. Any monomer on the left side of Figure 4 could, in principle, be combined with any monomer on the right side. Thus it is evident that a variety of polymers could be readily prepared by this method. Figure 5 illustrates four such polymers as examples. 

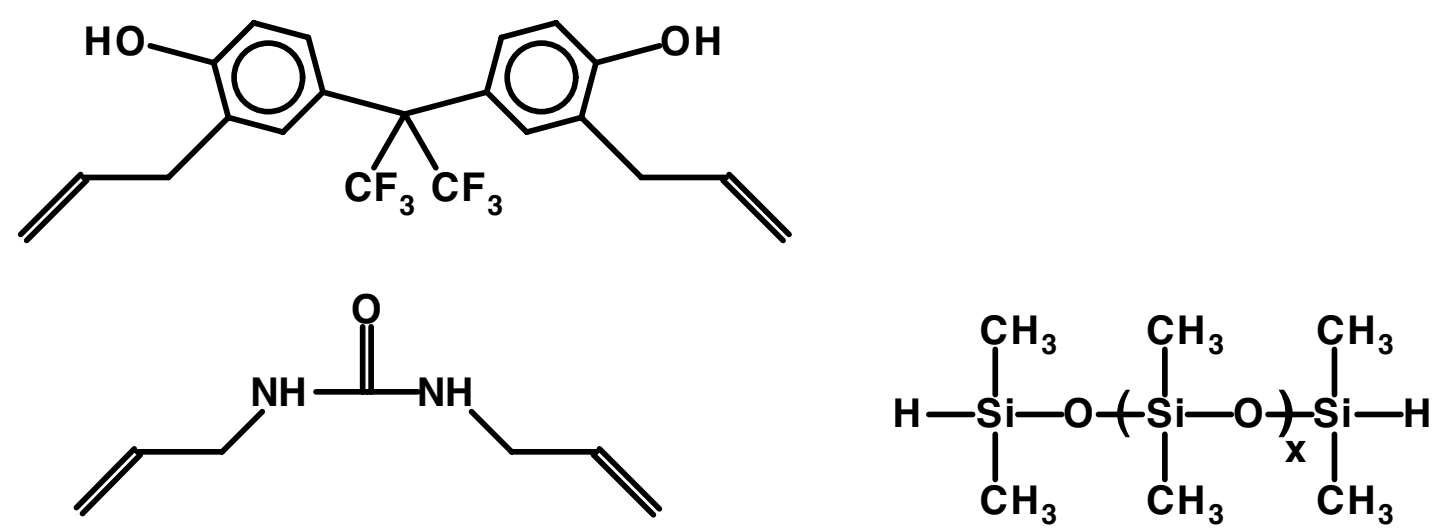<smiles>C=C[Si](C)(O[Si](C)(C=C)c1ccccc1)c1ccccc1</smiles>

$\mathrm{x}=0,1,4, \sim 80$ etc<smiles>c1ccc([SiH2]c2ccccc2)cc1</smiles><smiles>C=C[Si](C)(C)O[Si](C)(C)C=C</smiles>

Figure 4. The Chemical Structures of some Monomers Used for Making Carbosiloxane Polymers. The structure in the upper left is a bisphenol (BSP) monomer used for making BSP3 polymers and BSP-containing photopatterned films. 
Strong hydrogen bond acid<smiles>CCCCCCc1cc(C(C)(c2ccc(O)c(CCC[Si](C)(C)O[Si](C)(C)O[Si](C)(C)C)c2)C(F)(F)F)ccc1O</smiles>

Strong base, very dipolar UR3<smiles>CC[Si](C)(C)O[Si](C)(C)O[Si](C)(C)C</smiles>

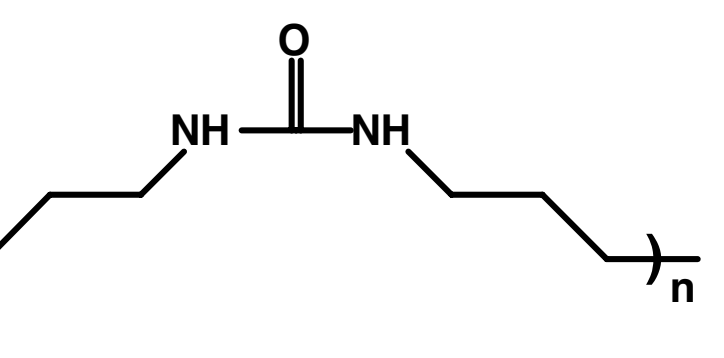

Polarizable CSPH<smiles>CC=C(c1ccccc1)[Si](C)(CC[Si](C)(CCC)C(F)(F)F)O[Si](C)(CCC(C)(C)C)c1ccccc1</smiles>

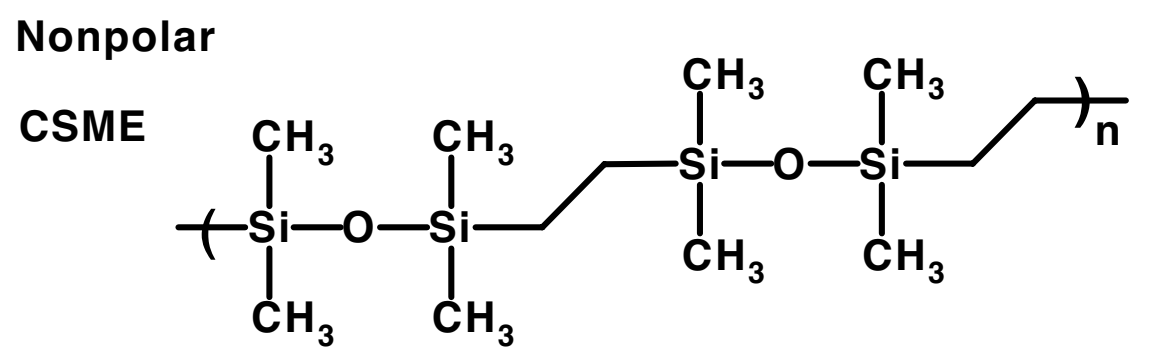

Figure 5. Selected Examples of Carbosiloxane Polymers Prepared for Selective Layers on Vapor Sensors, Illustrating a Wide Range of Chemical Structures and Interactive Properties 
Taken together, the polymers in Figure 5 can provide the chemical diversity that is desirable in small sensor arrays.[4, 12] Each of these polymers is designed to emphasize different properties and interactions. The methyl-substituted carbosiloxane polymer, dubbed CSME in the figure, is a nonpolar material that is good for sorbing aliphatic hydrocarbons. The phenyl-substituted polymer, $\mathrm{CSPH}$, provides greater polarizability than simple aliphatic groups in CSME. The polymer containing the urea group, UR3, was prepared in order to obtain a basic polymer for chemical sensor arrays. Urea groups are known to be very basic and dipolar. Finally, the polymer containing phenolic groups on a fluorinated structure, BSP3, was designed to interact as a hydrogen-bond acid with hydrogen-bond basic vapors.[35, 39, 40]

The last polymer (BSP3) illustrates a polymer made because no comparable commercial materials exist (i.e. strongly hydrogen bond acidic polymers with low glass transition temperatures for chemical sensing). Hydrogen-bond acidic materials are very desirable because there are many potential basic analytes one may wish to detect and recognize. Organophosphorus compounds in particular, such as nerve agents, are strong hydrogen bond bases. SAW devices coated with hydrogen-bond acidic polymers are among the best chemical microsensors for these toxic vapors. As a result, a number of synthetic efforts have focused on the development of polymers with these chemically interactive properties.[39-46] In the BSP3 polymer, the fluorinated bisphenol organic segments offer the desired chemical interactions while the siloxane segments are used to obtain desirable physical properties such as a low glass transition temperature.

The plots in Figure 6 and Figure 7 illustrate the performance of BSP3 compared to another hydrogen bond acidic polymer dubbed fluoropolyol (FPOL). FPOL has been used extensively in the past for organophosphorus vapor detection.[39, 40, 43, 44, 47-49] Figure 6 compares the response times of BSP3 and FPOL upon exposure to toluene at $4380 \mathrm{mg} / \mathrm{m}^{3}$. [39] Data points were collected every 2 seconds; it can be seen that BSP3 yields responses that reach steady state or near steady state in about 5 seconds under the test conditions. The measurement of response time is limited by the ability to deliver a step change in vapor concentration and this response time for BSP3 likely reflects vapor delivery rather than an intrinsic limitation in the speed of response. On the other hand the response by FPOL is clearly slower, indicating a slower diffusion into the bulk of the polymer and hence slower polymer/vapor equilibrium. 


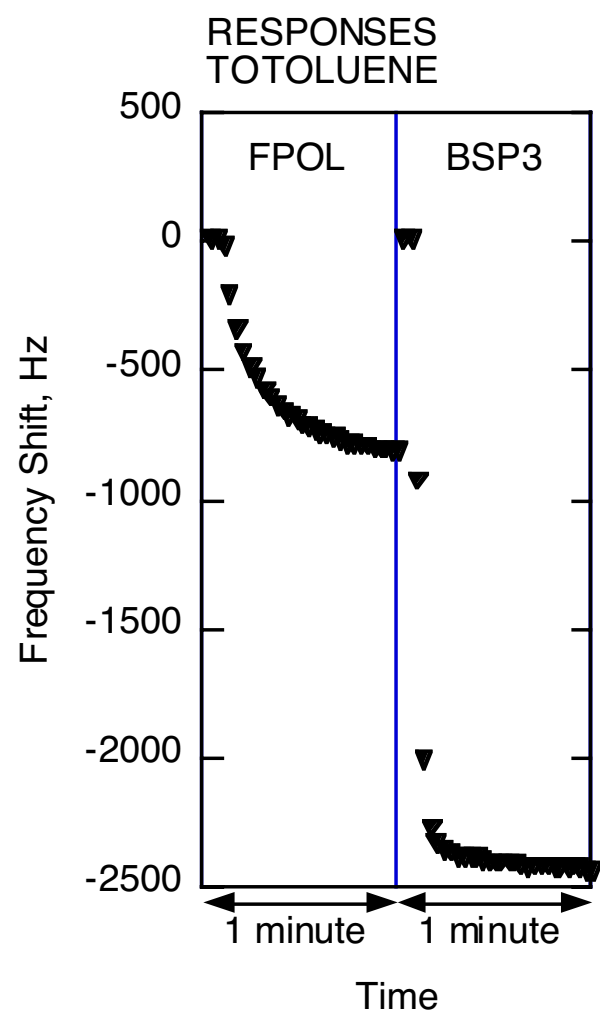

Figure 6. Comparison of the Response Time Behavior of Two Polymers, BSP3 and FPOL, to Toluene. Data points are two seconds apart.

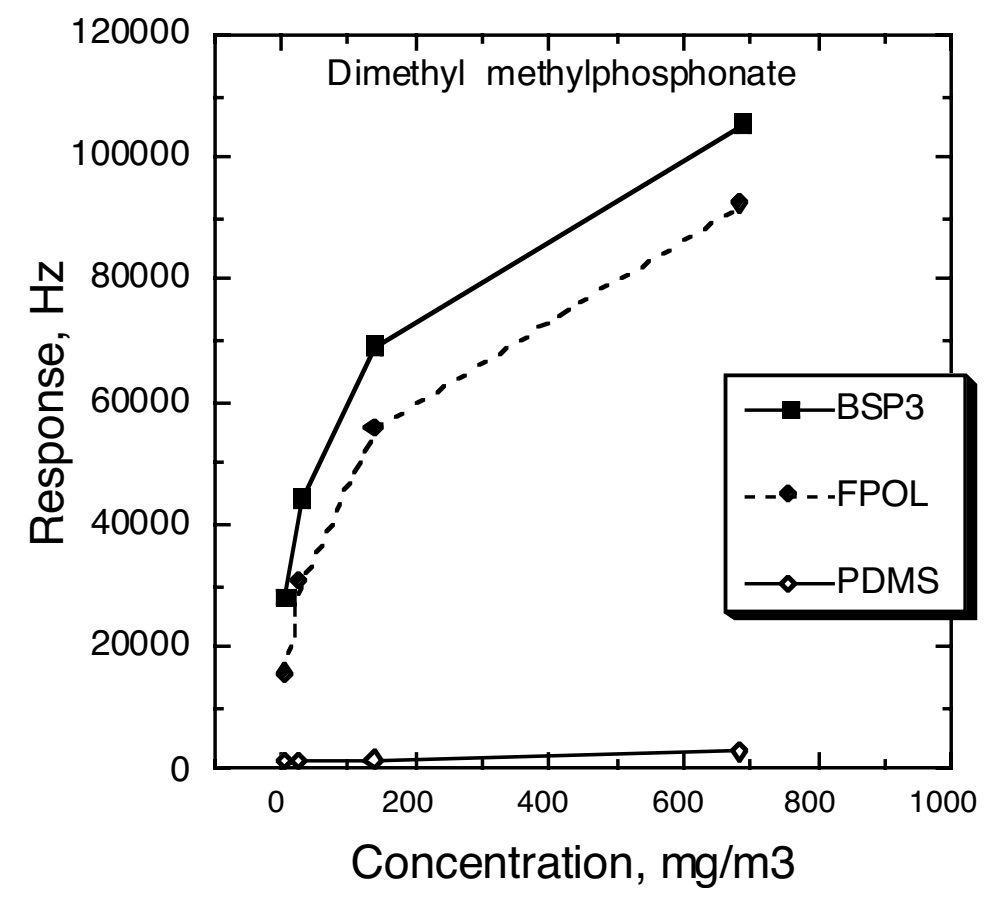

Figure 7. Calibration Curves Comparing the Sensitivities of Three Polymers (BSP3, FPOL, and PDMS) to dimethyl methylphosphonate (DMMP) 
Figure 7 plots the steady state response levels as a function of vapor concentration for dimethyl methylphosphonate (DMMP), a nerve agent simulant. [40] These are calibration curves and they demonstrate that BSP3 offers greater sensitivity than FPOL, especially at trace concentrations. Results for poly(dimethylsiloxane) (PDMS) are also shown; this polymer contains dimethyl(siloxane) linkages without the bisphenol segments as in BSP3. The very large difference in response between the BSP3 and the PDMS illustrates the importance of the incorporation of the hydrogen-bond acidic functional groups in order to obtain high sensitivity, and hence the success of the rational design approach. 


\subsection{Photopatterning Sorptive Polymeric Layers}

Polymeric materials are conventionally applied to sensor devices by solvent casting methods such as spin-coating, spray-coating, or drop-casting. As microsensor designs become smaller or more complex, it becomes increasingly important to be able to localize the sensing polymer on specific active regions of the device. In addition, array-on-a-chip sensor concepts require multiple different materials localized close to one another on the same chip without cross-contamination.

Photolithographic approaches represent an attractive approach for defining polymers in specific domains. However, conventional polymeric photoresists are not typically directly applicable as sensing layers since they are prepared with different properties than those desired for chemical sensing. Such photoresists are designed to be glassy materials that primarily serve as barrier layers and do not perform an active function on the final device. Often they have been etched away prior to device completion. A photopatternable sensing layer should be rubbery to promote rapid and reversible sorption, and it must perform an active function on the final device, and it must retain the desired chemical selectivity after completion of all the processing steps.

We set out to develop a method to directly photopattern selective layers in specific domains on a surface.[35] The overall approach is shown in Figure 8, where a polymer or prepolymer formulation is first solvent cast as a thin film on the surface. Exposing a selected region to ultraviolet (UV) light, either through a mask or by use of a focused light source from a micropattern generator, converts the exposed region to a material with less solubility in a "developing" solvent than the unexposed region. Upon washing with the developing solvent, only the exposed region remains on the surface.
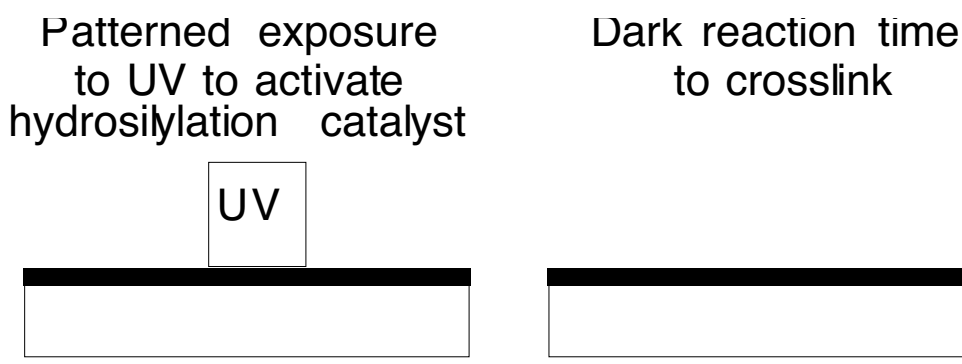
to crosslink

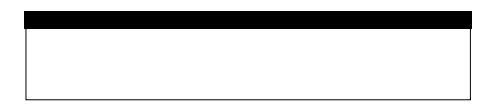

Figure 8. Schematic Diagram of the Photopatterning Process Where Those Domains Exposed to UV Light are Crosslinked and Retained on the Surface After Washing with a Developing Solvent, Whereas Those Domains Not Exposed Are Washed Away

The chemistry we selected to enable this approach was again hydrosilylation chemistry - this time for crosslinking reactions.[35] Films for photopatterning must contain a suitable polymer, a crosslinker, and a catalyst. The polymer or prepolymer is prepared with free $\mathrm{C}-\mathrm{C}$ double bonds, either pendant to the polymer chain or as chain ends. Figure 9 shows a few examples vinyl-modified organosiloxane polymers with vinyl groups providing the necessary $\mathrm{C}-\mathrm{C}$ double bonds. Crosslinkers are used that contain at least three $\mathrm{Si}-\mathrm{H}$ bonds available per crosslinker molecule. Figure 10 shows the structures of one polymeric and three molecular crosslinkers. Finally, the method requires a Pt catalyst that is inactive until exposed to light. Platinum(II) bis( $\beta$-diketonates) such as platinum(II) bis(acetylacetonate) $\left(\mathrm{Pt}(\mathrm{acac})_{2}\right)$ offer these properties. These compounds have been used as photoactivated hydrosilylation catalysts for solution reactions, polymerization of 
vinyldimethylsilane in solution, and for curing and patterning preceramic polymer films.[50-55] Photoactivated catalysis initiates hydrosilylation chemistry that crosslinks the film only in those regions exposed to the UV light.<smiles>C=C[Si](C)(C)O[Si](C)(C)O[Si](C)(C)C=C</smiles><smiles>C=C[Si](C)(C)O[Si](C)(C)O[Al](C)(C)C</smiles>

vinyl-modified OV-17

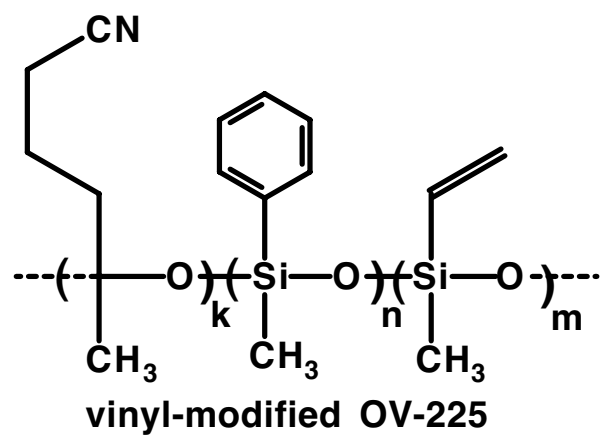

Figure 9. Repeat Unit Structures of some Vinyl-Containing Polyorganosiloxane Polymers Used for Making Photopatterned Films 

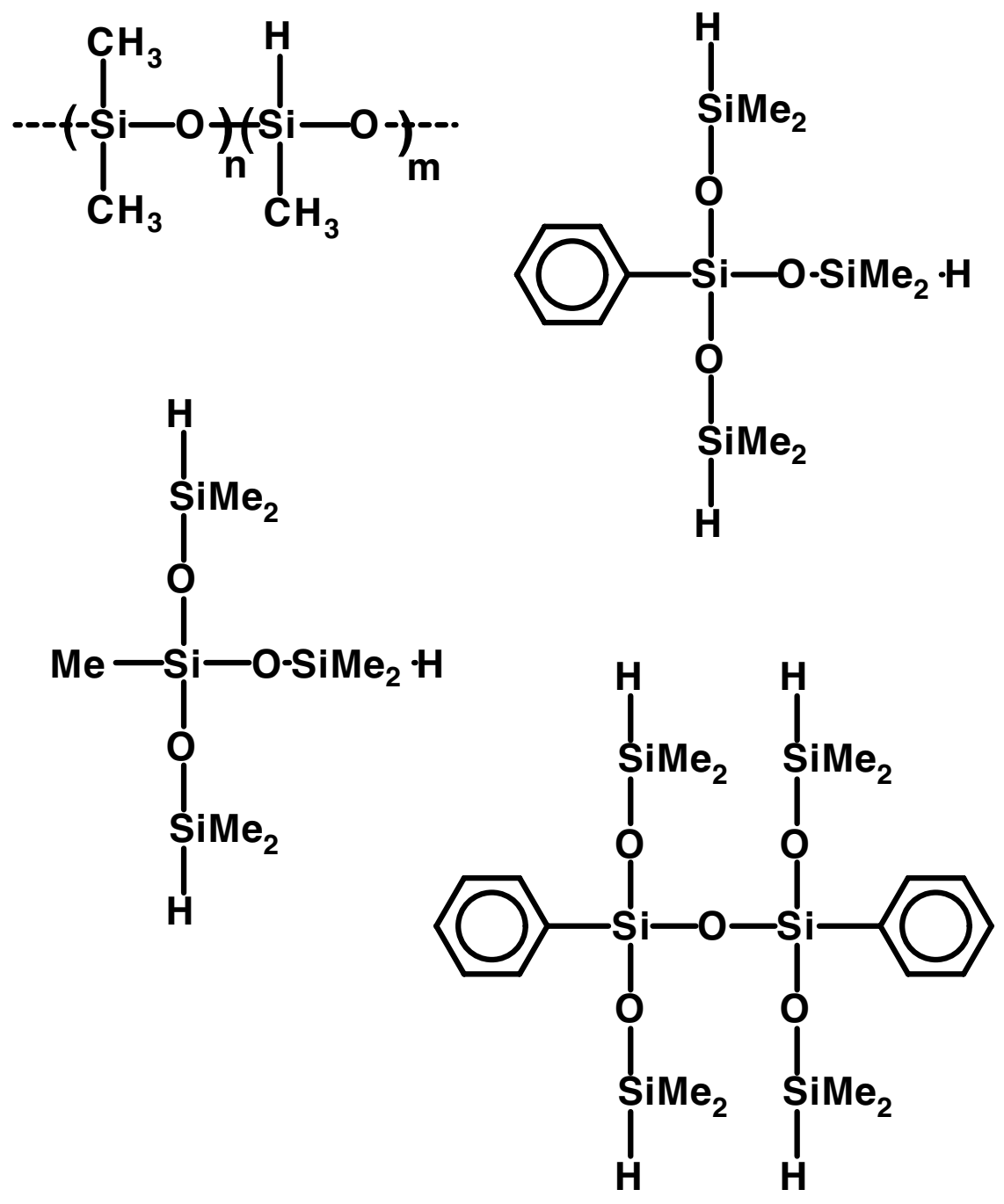

Figure 10. Structures of Some Crosslinkers Used for Making Photopatterned Films

We developed this chemistry to be useful for patterning functionalized siloxane and carbosiloxane polymers into specific domains on surfaces. Success requires that the crosslinker selected is miscible with the vinyl-functionalized polymer; the proportions of polymer and crosslinker must be appropriate for the vinyl content of the polymer; an adequate dark reaction time is necessary; and a suitable solvent must be found to dissolve away the unexposed polymer while leaving the exposed, crosslinked material intact on the surface. The last requirement can be particularly challenging since sorptive polymers that are useful for sensing also tend to swell in solvent, which stressed the film on the surface and can cause it to detach from the surface.

If so desired, the surface can first be functionalized with vinyl groups through the reaction of surface Si-OH groups with vinyl-silanizing reagents such as vinyltrimethoxysilane. Then the hydrosilylation chemistry will graft the polymer onto the surface at the same time that it is crosslinked. In some cases, this simultaneous grafting method can help to retain the pattern.

A diverse set of sorptive polymers can be photopatterned by this method. We exposed films using either a mask and an HTG 3A mask aligner, or a Research Devices photopattern generator. 
The light sources in both instruments are high pressure mercury lamps. , and representative formulations for mixing polymers and crosslinkers are given in Table 1. An oligomer synthesized for the preparation of photopatternable BSP3 polymers is shown in Figure 11. Images of patterned polymers on silicon wafers are shown in Figure 12.

Table 1. Formulations for Photopatterned Sorbent Films ${ }^{(a)}$

\begin{tabular}{|c|c|c|c|}
\hline $\begin{array}{l}\text { Polymer or } \\
\text { prepolymer } \\
\text { mixture }^{(b)}\end{array}$ & Crosslinker $^{(\mathrm{c})}$ & $\begin{array}{l}\text { Ratios } \\
\text { (by mass) }\end{array}$ & Properties \\
\hline $\begin{array}{l}\text { Vinyl-terminated } \\
\text { PDMS }\end{array}$ & $\begin{array}{l}\text { Poly(methylhydro)(dimethyl)- } \\
\text { siloxane }\end{array}$ & $25: 1$ & Nonpolar \\
\hline $\begin{array}{l}\text { Vinyl-modified } \\
\text { OV-17 }\end{array}$ & $\begin{array}{l}\text { Diphenyltetrakis(dimethylsiloxy)- } \\
\text { disiloxane }\end{array}$ & $\begin{array}{l}101: 1 \\
50: 1 \\
25: 1\end{array}$ & Polarizable \\
\hline $\begin{array}{l}\text { Vinyl-modified } \\
\text { OV-225 }\end{array}$ & $\begin{array}{l}\text { Diphenyltetrakis(dimethylsiloxy)- } \\
\text { disiloxane }\end{array}$ & $\begin{array}{l}200: 1 \\
101: 1 \\
50: 1\end{array}$ & Hydrogen-bond basic \\
\hline $\begin{array}{l}\text { BSP3 oligomer and } \\
\text { BSP monomer }\end{array}$ & $\begin{array}{l}\text { Diphenyltetrakis(dimethylsiloxy)- } \\
\text { disiloxane }\end{array}$ & $400: 100: 2.5$ & Hydrogen-bond acidic \\
\hline \multicolumn{4}{|c|}{$\begin{array}{l}\text { (a) The catalyst was typically } 2 \text { drops of } 0.5 \mathrm{M} \mathrm{Pt}(\mathrm{acac})_{2} \text { in dichloromethane added to (typically) } \\
2 \mathrm{~mL} \text { of polymer solution prior to spin casting. Exposure times ranged from seconds for simple } \\
\text { nonpolar PDMS films to experiments at } 2 \text { to } 10 \text { minutes exposure for the polar film materials. } \\
\text { Dark reaction times were minutes for the nonpolar films and } 18 \text { hours for the other more polar } \\
\text { films. Typical development solvents were xylene for the PDMS films and } \\
\text { dichloromethane:hexane }(2: 8 \mathrm{v} / \mathrm{v}) \text { for the polar films. } \\
\text { (b) Structure of the PDMS, OV-17, and OV-225 are shown in Figure } 9 \text {, the BSP3 oligomer structure } \\
\text { is shown in Figure } 11 \text {, and the BSP monomer is included in Figure } 4 \text {. } \\
\text { (c) Structures of the crosslinkers are shown in Figure } 10 \text {. }\end{array}$} \\
\hline
\end{tabular}<smiles>C[SiH](C)O[Si](C)(C)O[Si](C)(C)CCCc1cc(C(c2ccc(O)c(CCC[Si](C)(C)O[Si](C)(C)O[Si](C)(C)C)c2)(C(F)(F)F)C(F)(F)F)ccc1O</smiles>

Figure 11. Structure of the Si-H Terminated BSP3 Oligomer Used for Making Photopatterned BSP-Containing Films 


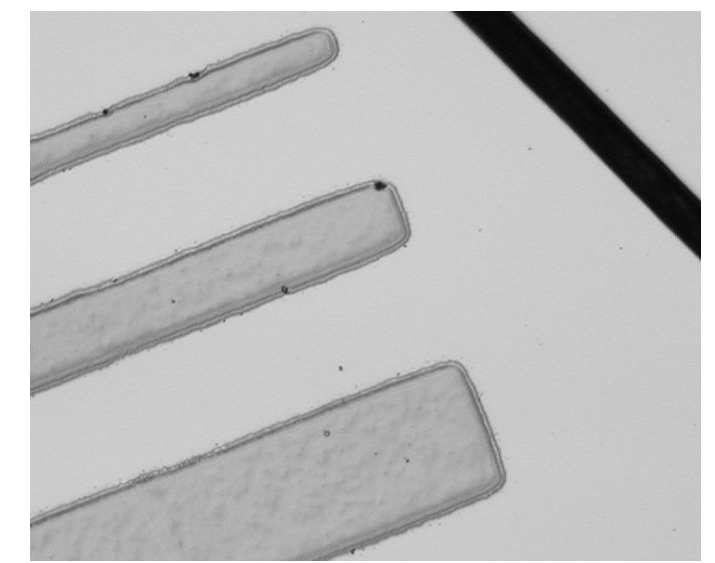

Figure $12 \mathrm{a}$

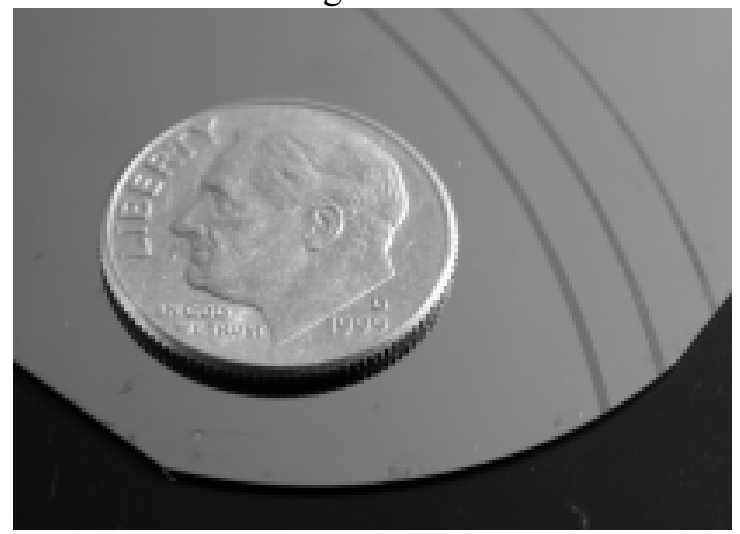

Figure 12c

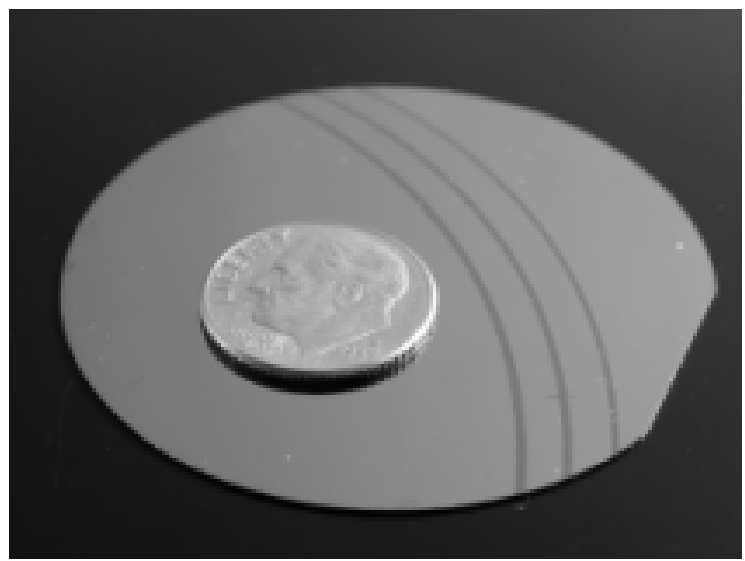

Figure $12 \mathrm{~b}$

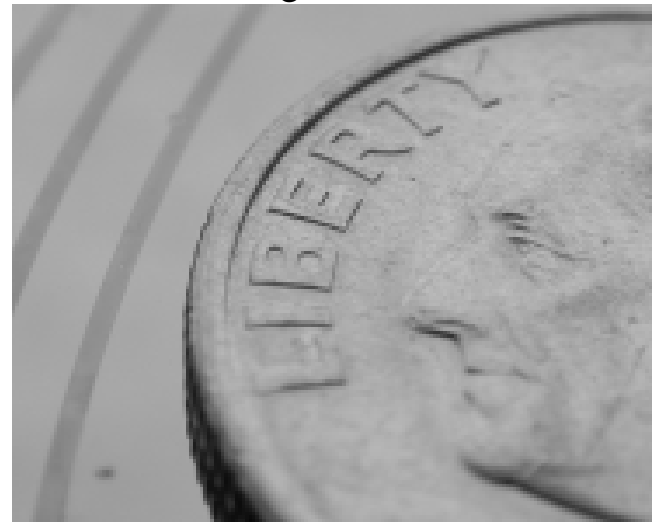

Figure 12d

Figure 12. Images of Photopatterned Polymer Films. a) Poly(dimethylsiloxane) photopatterned directly with a micropattern generator, where the central line is $\mathbf{1 0 0}$ microns in width and a human hair appearing as a black line has been included for scale. b) Photopatterned lines of $\mathrm{OV}-17$, where the lines were exposed for 2, 5 or 10 minutes and the mass ratio of polymer to crosslinker was 50:1. c) Photopatterned lines of $\mathrm{OV}-225$, where the lines were exposed for 2, 5 or 10 minutes and the mass ratio of polymer to crosslinker was 101:1. D) Close-up image of the photopatterned film related to the BSP3 polymer, prepared by simultaneously photopolymerizing and crosslinking the polymer as described in the text, shown next to a dime.

For example, nonpolar films can be photopatterned using vinyl-terminated PDMS and a polymeric crosslinker (see Figure 9 and Figure 10). We have photopatterned PDMS films from 50 $\mathrm{nm}$ to 5 micron in thickness by this method. In this example, both the vinyl-containing material and the Si-H containing crosslinker are polymeric.

Using vinyl modified OV-17 (see Figure 9), one can photopattern a layer that is more polarizable than PDMS. Using vinyl-modified OV-225 (see Figure 9), photopatterned films with basic cyano functional groups are obtained. Phenyl-containing molecular crosslinkers are used with the latter two polymers. The reactions with the vinyl-modified polyorganosiloxanes work with a range of polymer to crosslinker ratios. These examples combined a polymer with randomly distributed vinyl groups with a simple molecular silicon-hydride containing crosslinker. 
Photopatterned films were also developed that are related to the BSP3 polymer which contains bisphenol groups(see Figure 5 for the structure of the BSP3 polymer and Figure 4 for the bisphenol monomer used in the synthesis of BSP3). The photopatterning of these bisphenol-containing films involved photoactivated polymerization in addition to the usual photoactivated crosslinking. The prepolymer formulation included a $\mathrm{Si}-\mathrm{H}$ terminated BSP3 oligomer, the bisphenol monomer with its two C-C double bonds, a crosslinker molecule, and the photoactivated catalyst. The Si-H terminated oligomer, whose structure is shown in Figure 11, was prepared and isolated from the reaction of the bisphenol monomer in Figure 4 with excess of an $\alpha, \omega$-dihydrodimethylsiloxane (typically with three silicon atoms per monomer). This oligomer was mixed with the bisphenol monomer, a small amount of a phenyl-containing molecular crosslinker, and the $\mathrm{Pt}(\mathrm{acac})_{2}$ photoactivatable catalyst.

Photoactivated polymerization and crosslinking, followed by pattern development, was successful in producing photopatterned bisphenol-containing films as shown in Figure 12d. These reactions were done on a vinyl-modified silicon surface.

These photopatterning experiments demonstrate that photopatterned sorbent film materials can be made with a variety of functional groups and properties, as is required for sensor arrays. A number of them can be made starting with commercial materials. Alternatively, the carbosiloxane synthesis chemistry can be adapted readily to provide polymer and prepolymer formulations for photopatterning films with rationally designed chemical selectivity. 


\subsection{Conclusion}

Sorptive materials are useful for sensors and sensor arrays using a variety of transduction mechanisms. Prior work has shown that polymers can be selected or designed based on a rational understanding of vapor polymer interactions, which have been studied systematically using linear solvation energy relationships. The hydrosilylation chemistry described above provides a versatile approach to synthesizing rationally designed polymers with control over both the chemical and physical properties. We have now further shown that diverse sorptive polymers can be directly photopatterned into well defined domains on surfaces. 


\subsection{References}

[1] A. D'Amico and E. Verona, "SAW sensors," Sens. Actuators, vol. 17, pp. 55-66, 1989.

[2] J. F. Alder and J. J. McCallum, "Piezoelectric crystals for mass and chemical measurements," Analyst (London), vol. 108, pp. 1169-1189, 1983.

[3] C. G. Fox and J. F. Alder, "Surface acoustic wave sensors for atmospheric gas monitoring," Analyst(London), vol. 114, pp. 997-1004, 1989.

[4] J. W. Grate and M. H. Abraham, "Solubility interactions and the selection of sorbent coating materials for chemical sensors and sensor arrays," Sens. Actuators B, vol. 3, pp. 85-111, 1991.

[5] W. Gopel, "Chemical sensing, molecular electronics and nanotechnology: interface technologies down to the molecular scale," Sens. Actuators B, vol. 4, pp. 7-21, 1991.

[6] G. G. Guilbault and J. M. Jordan, "Analytical uses of piezoelectric crystals: a review," $C R C$ Critical Reviews in Analytical Chemistry, vol. 19, pp. 1-28, 1988.

[7] J. J. McCallum, "Piezoelectric devices for mass and chemical measurements: an update," Analyst (London), vol. 114, pp. 1173-1189, 1989.

[8] A. Mierzwinski and Z. Witkiewicz, "The application of piezoelectric detectors for investigations of environmental pollution," Environmental Pollution, vol. 57, pp. 181-198, 1989.

[9] M. S. Nieuwenhuizen and A. Venema, "Surface acoustic wave chemical sensors," Sens. Materials, vol. 5, pp. 261-300, 1989.

[10] F. C. J. M. Van Veggel, "Mass Sensors," Comprehensive Supramolecular Chemistry, vol. 10, pp. 171-185, 1996.

[11] K. J. Albert, N. S. Lewis, C. L. Schauer, G. A. Sotzing, S. E. Stitzel, T. P. Vaid, and D. R. Walt, "Cross-Reactive Chemical Sensor Arrays," Chem. Rev. (Washington, D. C.), vol. 100, pp. 25952626, 2000.

[12] J. W. Grate, "Acoustic Wave Microsensor Arrays for Vapor Sensing," Chemical Reviews, vol. 100, pp. 2627-2648, 2000.

[13] P. C. Jurs, G. A. Bakken, and H. E. McClelland, "Computational Methods for the Analysis of Chemical Sensor Array Data from Volatile Analytes," Chem. Rev. (Washington, D. C.), vol. 100, pp. 2649-2678, 2000.

[14] J. W. Grate, S. J. Martin, and R. M. White, "Acoustic Wave Microsensors, Part I," Anal. Chem., vol. 65, pp. 940A-948A, 1993.

[15] J. W. Grate, S. J. Martin, and R. M. White, "Acoustic Wave Microsensors, Part II," Anal. Chem., vol. 65, pp. 987A-996A, 1993. 
[16] J. W. Grate and G. C. Frye, "Acoustic Wave Sensors," in Sensors Update, vol. 2, H. Baltes, W. Goepel, and J. Hesse, Eds. Weinheim: VSH, 1996, pp. 37-83.

[17] D. S. Ballantine, R. M. White, S. J. Martin, A. J. Ricco, E. T. Zellers, G. C. Frye, and H. Wohltjen, Acoustic Wave Sensors. Theory, Design, and Physico-Chemical Applications. New York: Academic Press, 1997.

[18] M. H. Thompson and D. C. Stone, Surface-Launched Acoustic Wave Sensors : Chemical Sensing and Thin-Film Characterization. New York: John Wiley and Sons, 1997.

[19] B. Lundberg and B. Sundqvist, "Resistivity of a composite conducting polymer as a function of temperature, pressure, and environment: applications as a pressure and gas concentration transducer," J. Appl. Phys., vol. 60, pp. 1074-1079, 1986.

[20] P. Talik, M. Zabkowska-Waclawek, and W. Waclawek, "Sensing properties of the CB-PCV composites for chlorinated hydrocarbon vapors," J. Mater. Sci., vol. 27, pp. 6807-6810, 1992.

[21] M. C. Lonergan, E. J. Severin, B. J. Doleman, S. A. Beaber, R. H. Grubbs, and N. S. Lewis, "Array-based vapor sensing using chemically sensitive carbon black-polymer resistors," Chem. Mater., vol. 8, pp. 2298-2312, 1996.

[22] B. J. Doleman, M. c. Lonergan, E. J. Severin, T. P. Vaid, and N. S. Lewis, "Quantitative Study of the resolving power of arrays of carbon black-polymer composites in various vapor-sensing tasks," Anal. Chem., vol. 70, pp. 4177-4190, 1998.

[23] E. J. Severin and N. S. Lewis, "Relationships among Resonant Freqency Changes on a Coated Quartz Crystal Microbalance, Thickness Changes, and Resistance Responses of Polymer-Carbon Black Composite Chemiresistors," Anal. Chem., vol. 72, pp. 2008-2015, 2000.

[24] E. J. Severin, B. J. Doleman, and N. S. Lewis, "An Investigation of the Concentration Dependence and Response to Analyte Mixtures of Carbon Black/Insulating Organic Polymer Composite Vapor Detectors," Anal. Chem., vol. 72, pp. 658-668, 2000.

[25] T. A. Dickinson, J. White, J. S. Kauer, and D. R. Walt, "A chemical-detecting system based on a cross-reactive optical sensor array," Nature (London), vol. 382, pp. 697-700, 1996.

[26] J. White, J. S. Kauer, T. A. Dickinson, and D. R. Walt, "Rapid Analyte Recognition in a Device Based on Optical Sensors and the Olfactory System," Anal. Chem., vol. 68, pp. 2191-2202, 1996.

[27] K. L. Michael, L. C. Taylor, S. L. Schultz, and D. R. Walt, "Randomly Ordered Addressable High-Density Optical Sensor Arrays," Anal. Chem., vol. 70, pp. 1242-1248, 1998.

[28] T. A. Dickinson, K. L. Michael, J. S. Kauer, and D. R. Walt, "Convergent, Self-Encoded Bead Sensor Arrays in the Design of an Artificial Nose," Anal. Chem., vol. 71, pp. 2192-2198, 1999.

[29] L. M. Webber, K. H. Karmarker, and G. G. Guilbault, Anal. Chem. Acta, vol. 97, pp. 29, 1978. 
[30] G. C. Frye, S. J. Martin, and A. J. Ricco, "Monitoring diffusion in real time in thin polymer films using SAW devices," Sens. \& Materials, vol. 1, pp. 335-357, 1989.

[31] N. Barie, M. Rapp, and H. J. Ache, "UV crosslinked polysiloxanes as new coating materials for SAW devices with high long-term stability," Sens. Actuators, B, vol. B46, pp. 97-103, 1998.

[32] A. Hierlemann, U. Wiemar, G. Kraus, G. Gauglitz, and W. Goepel, "Environmental chemical sensing using quartz microbalance sensor arrays: application of multicomponent analysis techniques," Sens. Mater., vol. 7, pp. 179-189, 1995.

[33] J. Park, W. A. Groves, and E. T. Zellers, "Vapor Recognition with Small Arrays of PolymerCoated Microsensors. A Comprehensive Analysis," Anal. Chem., vol. 71, pp. 3877-3886, 1999.

[34] J. W. Grate, M. H. Abraham, and R. A. McGill, "Sorbent Polymer Coatings for Chemical Sensors and Arrays," in Handbook of Biosensors: Medicine, Food, and the Environment, E. KressRogers and S. Nicklin, Eds. Boca Raton, FL, USA: CRC Press, 1996, pp. 593-612.

[35] J. W. Grate, S. N. Kaganove, and D. A. Nelson, "Carbosiloxane Polymers for Chemical Sensors," Chemical Innovations, vol. 30(11), pp. 29-37, 2000.

[36] J. W. Grate and S. N. Kaganove, "Hydrosilylation: A Versatile Reaction for Polymer Synthesis," Polymer News, vol. 24, pp. 149-155, 1999.

[37] I. Ojima, "The hydrosilylation reaction," in The Chemistry of Organic Silicon Compounds, S. Patai and Z. Rappoport, Eds. New York: John Wiley and Sons Ltd, 1989, pp. 1479-1526.

[38] B. Marciniec, "Comprehensive Handbook on Hydrosilylation,". Oxford: Pergamon Press, 1992.

[39] J. W. Grate, S. N. Kaganove, and S. J. Patrash, "Hydrogen-Bond Acidic Polymers for Surface Acoustic Wave Vapor Sensors and Arrays," Anal. Chem., vol. 71, pp. 1033-1040, 1999.

[40] J. W. Grate, S. N. Kaganove, S. J. Patrash, R. Craig, and M. Bliss, "Hybrid Organic/Inorganic Copolymers with Strongly Hydrogen-Bond Acidic Properties for Acoustic Wave and Optical Sensors," Chem. Mater., vol. 9, pp. 1201-1207, 1997.

[41] M. H. Abraham, I. Hamerton, J. B. Rose, and J. W. Grate, "Hydrogen bonding Part 18. Gas-liquid chromatographic measurements for the design and selection of some hydrogen bond acidic phases suitable for use as coatings on piezoelectric sorption detectors," J. Chem. Soc. Perkin Trans. 2, pp. 1417-1423, 1991.

[42] A. W. Snow, L. G. Sprague, R. L. Soulen, J. W. Grate, and H. Wohltjen, "Synthesis and evaluation of hexafluorodimethylcarbinol functionalized polymers as microsensor coatings," $J$. Appl. Poly. Sci., vol. 43, pp. 1659-1671, 1991.

[43] M. H. Abraham, J. Andonian-Haftvan, C. M. Du, V. Diart, G. Whiting, J. W. Grate, and R. A. McGill, "Hydrogen Bonding. XXIX. The characterization of fourteen sorbent coatings for 
chemical microsensors using a new solvation equation," J. Chem. Soc., Perkin Trans. 2, pp. 369$378,1995$.

[44] J. W. Grate, A. Snow, D. S. Ballantine, H. Wohltjen, M. H. Abraham, R. A. McGill, and P. Sasson, "Determination of partition coefficients from surface acoustic wave vapor sensor responses and correlation with gas-liquid chromatographic partition coefficients," Anal. Chem., vol. 60, pp. 869-875, 1988.

[45] J. W. Barlow, P. E. Cassidy, D. R. Lloyd, C. J. You, Y. Chang, P. C. Wong, and J. Noriyan, "Polymer sorbents for phosphorus esters: II. Hydrogen bond driven sorption in fluoro-carbinol substituted polystyrene," Polym. Eng. Sci., vol. 27, pp. 703-715, 1987.

[46] Y. Chang, J. Noriyan, D. R. Lloyd, and J. W. Barlow, "Polymer sorbents for phosphorus esters: I. Selection of polymers by analog calorimetry," Polym. Eng. Sci., vol. 27, pp. 693, 1987.

[47] J. W. Grate, S. L. Rose-Pehrsson, D. L. Venezky, M. Klusty, and H. Wohltjen, "Smart sensor system for trace organophosphorus and organosulfur vapor detection employing a temperaturecontrolled array of surface acoustic wave sensors, automated sample preconcentration, and pattern recognition," Anal. Chem., vol. 65, pp. 1868-1881, 1993.

[48] S. L. Rose-Pehrsson, J. W. Grate, D. S. Ballantine, and P. C. Jurs, "Detection of hazardous vapors including mixtures using pattern recognition analysis of responses from surface acoustic wave devices," Anal. Chem., vol. 60, pp. 2801-2811, 1988.

[49] D. S. Ballantine, S. L. Rose, J. W. Grate, and H. Wohltjen, "Correlation of surface acoustic wave device coating responses with solubility properties and chemical structure using pattern recognition," Anal. Chem., vol. 58, pp. 3058-3066, 1986.

[50] G. D. Salvi, "The photochemical behavior of the group 10(II) bis(b-diketonates) and the use of platinum(II) bis(b-diketonates) as photoactivated hydrosilation catalysts," Ph.D. Thesis, Northwestern Univ., Evanston, IL, USA., 1993, pp. 324 pp.

[51] F. D. Lewis, and G. D. Salvi, "Platinum(II) Bis(b-diketonates) as Photoactivated Hydrosilation Catalysts," Inorg. Chem., vol. 34, pp. 3182-9, 1995.

[52] B. E. Fry and D. C. Neckers, "Rapid Photoactivated Hydrosilation Polymerization of Vinyldimethylsilane," Macromolecules, vol. 29, pp. 5306-5312, 1996.

[53] B. E. Fry, A. Guo, and D. C. Neckers, "Photoactivated hydrosilation curing of a ceramic precursor: crosslinking and pyrolysis of branched oligo[(methylsilylene)methylene]," $J$. Organomet. Chem., vol. 538, pp. 151-162, 1997.

[54] B. E. Fry, "Photoactivated hydrosilation polymerization and the synthesis, crosslinking and pyrolysis of oligocarbosilane ceramic precursors (platinum complex)," , Ph.D. Thesis, Bowling Green State Univ., Bowling Green, OH, USA., 1996, pp. 130 pp.

[55] A. Guo, B. E. Fry, and D. C. Neckers, "Highly Active Visible-Light Photocatalysts for Curing a Ceramic Precursor," Chem. Mater., vol. 10, pp. 531-536, 1998. 


\section{Appendix A}

\section{Detailed Experimental Procedures}




\section{Appendix A: Detailed Experimental Procedures}

Materials. Solvents and reagents obtained from commercial sources were used as received. 2,2Bis(3-allyl-4-hydroxyphenyl)hexafluoropropane was obtained from Organic Consultants, Inc., Eugene, Oregon. This material was stored at room temperature as a dichloromethane solution. The BSP-3 trimer (DN-96) was prepared at PNNL. Phenylmethylsiloxane polymers were obtained from Ohio Valley Specialty Chemical (OV-17 and OV-225). The PDMS was obtained from Gelest, Inc (DMS-V31)as was 1,3-diphenyl-1,1,3,3-tetrakis(dimethylsiloxy)disiloxane and HMS-301. The platinum catalyst was obtained from Aldrich Chemical Co.

Photopatterning Equipment. Promising materials were applied to silicon wafers by spin coating and exposed to ultraviolet light in a HTG 3A mask aligner. Designed for large area proximity printing, the HTG unit expands the light from a high pressure, mercury lamp; the final beam intensity, between $300-400 \mathrm{~nm}$, is about $10 \mathrm{~mW} / \mathrm{cm}^{2}$. The light is a uniform and collimated $20 \mathrm{~cm}$ diameter beam which is patterned through a photomask before striking the coated wafer. The photomask is an iron oxide coated glass plate made selectively transparent to the ultraviolet light by etching away regions of the iron mask. For these experiments, the opening in the iron mask was a 280 micron wide line.

The Research Devices micropattern generator uses an Oriel 500 watt high pressure mercury lamp to illuminate photosensitive material through a Nikon microscope. This light source has strong irradiance between 300 and $450 \mathrm{~nm}$. The beam is collimated, then shaped by focusing through a variable rectangular aperture and sized by focusing through the microscope objective. Rectangular spots between 5 and 500 microns in either dimension are possible. The UV light power at the sample is about $4000 \mathrm{~mW} / \mathrm{cm} 2$. Patterns are created by opening and closing a high speed shutter as the precision stage is translated around a CAD drawing. The aperture shape also changes as needed to pattern the various features.

Film Photopatterning Technique - General Procedure. Silicon wafers $(5.08 \mathrm{~cm}$ diameter) were plasma cleaned within a PDC-3XG Plasma Cleaner (Harrick Scientific Corp.) for 30 min., then reacted with coupling agents (silanes), if necessary, as described below. The polymer or prepolymer solution was evenly applied to the silicon wafers and was spin cast on the silicon wafers at $3000 \mathrm{rpm}$ for 30 seconds. The coated wafer was exposed using either a micropattern generator or a mask aligner for varying times). After exposure, the wafer was either processed with solvent immediately (for example polydimethylsiloxanes) or was stored in the dark for 18 hours before solvent processing. Developing times were usually less than three minutes.

Surface Modification Techniques. Surfaces were plasma cleaned prior to surface modification. The substrates were placed within a $250 \mathrm{~mL}$ reaction kettle (Teflon ${ }^{\circledR}$ gasket), equipped with a condenser and nitrogen source. To the kettle was added $0.3 \mathrm{~g}$ of coupling agent in $70 \mathrm{~mL}$ of HPLCgrade acetone. The silane coupling agents examined were triethoxysilane and triethoxyvinylsilane. The solution was stirred at approximately $50^{\circ} \mathrm{C}$ for $48 \mathrm{~h}$. The substrate was removed form the cooled solution, washed with clean acetone, dried under a nitrogen stream, and stored in a drying chamber until use. 


\section{Triad Containing Bisphenol-Monomer and Siloxane}

The Bisphenol-A monomer terminated with hexamethyltrisiloxane groups (Triad) was prepared in the following manner: 2,2-bis(3-allyl-4-hydroxyphenyl)hexafluoropropane $(2.85 \mathrm{~g}, 0.0068 \mathrm{~mol})$ was added to a magnetically stirred solution of 1,1,3,3,5,5-hexamethyltrisiloxane $(14.3 \mathrm{~g}, 0.068$ mole) in $100 \mathrm{~mL}$ of toluene. To this solution was added two drops of platinum-1,3-

divinyltetramethyldisiloxane in xylene. The solution was heated to $100^{\circ} \mathrm{C}$ for $18 \mathrm{~h}$. The solution was cooled, stirred with activated carbon for $24 \mathrm{~h}$, and filtered with vacuum and syringe filter techniques. The solvent and excess hexamethyldisiloxane were removed by distillation. Vacuum distillation was unsuccessful due to gellation of the product above $150^{\circ} \mathrm{C}$. The yield of straw-colored oil (Triad) accounted for $5.4 \mathrm{~g}$ or $95 \%$, based upon a presumed 3-unit product of $\mathrm{M}_{\mathrm{w}}, 833$.

Preparative HPLC was used in an attempt to isolate the major products; the composition contained within this major fraction was $t^{\prime}{ }_{R}$ (area\%): $3.5(3.6 \%), 4.3(8.3 \%), 4.8(2.5 \%), 8.7(2.8 \%), 9.7$

(51.1\%), $10.2(21.9 \%), 12.0(4.2 \%) .{ }^{13} \mathrm{C} \mathrm{NMR}\left(\mathrm{CDCl}_{3}\right)$ 154.1, 133.2, 132.6, 129.6, 128.4, 128.3, $128.1,125.8,125.7,114.9,114.8,125.1$ (q), 63.9 (m), 33.7, 33.5, 23.8, 23.2, 18.2, 18.0, 1.4, 1.2, $0.4,-0.1$. FTIR indicated a strong absorbance at $2127 \mathrm{~cm}^{-1}$ which is indicative of Si-H. 


\section{Example: Poly(dimethylsiloxane) - Nonpolar}

The following example shows the photopatterning of a nonpolar sorbent material consisting of polydimethylsiloxane. Two polymers are mixed with catalyst. One polymer, DMS-V31 consists of polydimethylsiloxane with terminal vinyl groups. The other polymer, HMS-310, has silicon hydride groups distributed along the chain. Thus, in this example, both the vinyl containing polymer and the Si-H containing crosslinker are polymeric.

The following materials were used and combined in the proportions indicated.

DMS-V31 (vinyl-terminated dimethylsiloxane, MW, 28,000)

HMS-301 (hydromethyldimethylsiloxane, MW, 2000 and 25\% MeSi-H)

Pt (acac) $)_{2}$ As the catalyst

\section{Composition Amounts:}

0.057 g DMS-V31

0.020 g HMS-301

2 drops of $0.05 \mathrm{M} \mathrm{Pt}(\mathrm{acac})_{2}$ in dichloromethane

$0.5 \mathrm{~mL}$ dichloromethane as solvent

A silicon wafer was plasma cleaned and a film was coated onto the surface by spin-coating at 3000 rpm, using the solution composition just described above.

\section{Surface Preparation:}

Plasma cleaned for $30 \mathrm{~min}$

\section{Spin Cast Conditions:}

$3000 \mathrm{rpm}$

The wafer was exposed using the mask aligner system and immediately washed with xylene to develop the pattern. Individual lines were exposed for $60,120,140,180$, and $300 \mathrm{~s}$. The solvent removed the unexposed material leaving a pattern of lines corresponding to the 120,140,180, and $300 \mathrm{~s}$ exposures. The line exposed only for $60 \mathrm{~s}$ did not remain after developing the pattern with the xylene solvent wash.

\section{Masking Conditions:}

Individual line exposure times at $60,120,140,180$, and $300 \mathrm{~s}$

Dark reaction time was: immediate solvent development.

Wash solvent was xylene.

\section{Comments:}

$60 \mathrm{~s}$ line was removed by solvent, but others remained intact. No improvement was noted if the polymer solution was applied to triethoxyvinylsilane treated Si-wafer. 


\section{Example: OV-17 101:1}

The following example shows the photopatterning of a polysiloxane that contains phenyl substituents. These increase the polarizability of a sensing polymer relative to polydimethyl siloxanes. The polymer in this example is $50 \%$ phenyl substituted and it contains $1 \%$ vinyl substituents randomly distributed along the chain. The crosslinker is a simple tetrafunctional crosslinker, meaning it has four silicon hydride groups. Thus this example combined a polymer with randomly distributed vinyl groups with a simple molecular silicon-hydride containing crosslinker.

\section{Phenyl-Substituted Polysiloxane}

OV-17 (50\% phenyl, 1 mole\% random vinyl, Mw, 4000)

Si-H Cross-linker

1,3-diphenyl-1,1,3,3-tetrakis(dimethylsiloxy)disiloxane, Mw, 527

\section{Catalyst}

$\operatorname{Pt}(\text { acac })_{2}$

\section{Composition Amounts}

$0.22 \mathrm{~g} \mathrm{OV}-17$ (equates to $1.7 \times 10^{-5}$ equivalents of vinyl)

$0.0022 \mathrm{~g}\left(4.2 \times 10^{-6} \mathrm{~mole}\right)$ diphenyltetrakis(dimethylsiloxy)disiloxane

2 drops of $0.5 \mathrm{M} \mathrm{Pt}(\mathrm{acac})_{2}$ in dichloromethane

$1.7 \mathrm{~mL}$ dichloromethane added

Mass ratio of polymer: cross-linker, 101:1

The silicon wafer was cleaned with ozone-uv (UVO cleaner, Jelight). A film was coated onto the wafer surface by spin-coating at $3000 \mathrm{rpm}$ using the solution composition described above.

\section{Surface Preparation}

Silicon wafer

Ozone-uv treated for 2 minutes.

\section{Spin Cast Conditions}

$3000 \mathrm{rpm}$

\section{Photopatterning Conditions}

The wafer was exposed to ultraviolet light using the mask aligner system.

Individual arc-lines were exposed for 2, 5, and 10 minutes.

Dark reaction time was $18 \mathrm{hrs}$

Solvent development with dichloromethane: hexane $(2: 8 \mathrm{v} / \mathrm{v})$.

\section{Comments}

The solvent removed the unexposed material leaving a pattern of lines corresponding to all three lines (complete lines for 2, 5 and 10 minutes). 


\section{Example: OV-17 50:1}

The following example shows the photopatterning of a polysiloxane that contains phenyl substituents. These increase the polarizability of a sensing polymer relative to polydimethyl siloxanes. The polymer in this example is $50 \%$ phenyl substituted and it contains $1 \%$ vinyl substituents randomly distributed along the chain. The crosslinker is a simple tetrafunctional crosslinker, meaning it has four silicon hydride groups. Thus this example combined a polymer with randomly distributed vinyl groups with a simple molecular silicon-hydride containing crosslinker.

\section{Phenyl-Substituted Polysiloxane}

OV-17 (50\% phenyl, 1 mole\% random vinyl, Mw, 4000)

Si-H Cross-linker

1,3-diphenyl-1,1,3,3-tetrakis(dimethylsiloxy)disiloxane, Mw, 527

\section{Catalyst}

$\operatorname{Pt}(\text { acac })_{2}$

\section{Composition Amounts}

$0.23 \mathrm{~g} \mathrm{OV}-17$ (equates to $1.7 \times 10^{-5}$ equivalents of vinyl)

$0.0046 \mathrm{~g}\left(8.8 \times 10^{-6}\right.$ mole $)$ diphenyltetrakis(dimethylsiloxy)disiloxane

2 drops of $0.5 \mathrm{M} \mathrm{Pt}(\mathrm{acac})_{2}$ in dichloromethane

$1.8 \mathrm{~mL}$ dichloromethane added

Mass ratio of polymer: cross-linker, 50:1

The silicon wafer was cleaned with ozone-uv. A film was coated onto the wafer surface by spincoating at $3000 \mathrm{rpm}$ using the solution composition described above.

\section{Surface Preparation}

Silicon wafer

Ozone-uv treated for 2 minutes.

\section{Spin Cast Conditions}

$3000 \mathrm{rpm}$

\section{Photopatterning Conditions}

The wafer was exposed to ultraviolet light using the mask aligner system.

Individual arc-lines were exposed for 2, 5, and 10 minutes.

Dark reaction time was $18 \mathrm{hrs}$

Solvent development with dichloromethane: hexane $(2: 8 \mathrm{v} / \mathrm{v})$.

\section{Comments}

The solvent removed the unexposed material leaving a pattern of lines corresponding to all three lines (complete lines for 2, 5 and 10 minutes). 


\section{Example: OV-17 25:1}

The following example shows the photopatterning of a polysiloxane that contains phenyl substituents. These increase the polarizability of a sensing polymer relative to polydimethyl siloxanes. The polymer in this example is $50 \%$ phenyl substituted and it contains $1 \%$ vinyl substituents randomly distributed along the chain. The crosslinker is a simple tetrafunctional crosslinker, meaning it has four silicon hydride groups. Thus this example combined a polymer with randomly distributed vinyl groups with a simple molecular silicon-hydride containing crosslinker.

\section{Phenyl-Substituted Polysiloxane}

OV-17 (50\% phenyl, 1 mole\% random vinyl, Mw, 4000)

Si-H Cross-linker

1,3-diphenyl-1,1,3,3-tetrakis(dimethylsiloxy)disiloxane, Mw, 527

\section{Catalyst}

$\operatorname{Pt}(\text { acac })_{2}$

\section{Composition Amounts}

$0.29 \mathrm{~g} \mathrm{OV}-17$ (equates to $2.2 \times 10^{-5}$ equivalents of vinyl)

$0.012 \mathrm{~g}\left(2.2 \times 10^{-5}\right.$ mole) diphenyltetrakis(dimethylsiloxy)disiloxane

2 drops of $0.5 \mathrm{M} \mathrm{Pt}(\mathrm{acac})_{2}$ in dichloromethane

$2.3 \mathrm{~mL}$ dichloromethane added

Mass ratio of polymer: cross-linker, 25:1

The silicon wafer was cleaned with ozone-uv. A film was coated onto the wafer surface by spincoating at $3000 \mathrm{rpm}$ using the solution composition described above.

\section{Surface Preparation}

Silicon wafer

Ozone-uv treated for 2 minutes.

\section{Spin Cast Conditions}

$3000 \mathrm{rpm}$

\section{Photopatterning Conditions}

The wafer was exposed to ultraviolet light using the mask aligner system.

Individual arc-lines were exposed for 2, 5, and 10 minutes.

Dark reaction time was $18 \mathrm{hrs}$

Solvent development with dichloromethane: hexane $(2: 8 \mathrm{v} / \mathrm{v})$.

\section{Comments}

The solvent removed the unexposed material leaving a pattern of lines corresponding to all three lines (complete lines for 2, 5 and 10 minutes). 


\section{Example: OV-225 200:1}

The following example shows the photopatterning of a polysiloxane that contains cyanosubstituents. These increase the dipolarity and basicity of a sensing polymer relative to polydimethyl siloxanes. The polymer in this example is $50 \%$ cyanopropylmethyl substituted and $50 \%$ phenylmethyl substituted, and it contains $1 \%$ vinyl substituents randomly distributed along the chain. The crosslinker is a simple tetrafunctional crosslinker, meaning it has four silicon hydride groups. Thus this example combined a polymer containing very polar functionalities and randomly distributed vinyl groups with a simple molecular silicon-hydride containing crosslinker.

The following materials were used and combined in the proportions indicated.

\section{Cyano-Substituted Polysiloxane}

OV-225 (cyanopropylmethylphenylsiloxane, 1 mole\% random vinyl, Mw, 8000)

Si-H Cross-linker

1,3-diphenyl-1,1,3,3-tetrakis(dimethylsiloxy)disiloxane, Mw, 527

\section{Catalyst}

$\operatorname{Pt}(\mathrm{acac})_{2}$

\section{Composition Amounts}

$0.28 \mathrm{~g} \mathrm{OV}-225$ (equates to $2.1 \times 10^{-5}$ equivalents of vinyl)

$0.0014 \mathrm{~g}\left(2.7 \times 10^{-6}\right.$ mole $)$ diphenyltetrakis(dimethylsiloxy)disiloxane

2 drops of $0.5 \mathrm{M} \mathrm{Pt}(\mathrm{acac})_{2}$ in dichloromethane

$2.2 \mathrm{~mL}$ dichloromethane added

Mass ratio of polymer: cross-linker, 200:1

The silicon wafer was cleaned with ozone-uv. A film was coated onto the wafer surface by spincoating at $3000 \mathrm{rpm}$ using the solution composition described above.

\section{Surface Preparation}

Silicon wafer

Ozone-uv treated for 2 minutes.

\section{Spin Cast Conditions}

$3000 \mathrm{rpm}$

\section{Photopatterning Conditions}

The wafer was exposed to ultraviolet light using the mask aligner system.

Individual arc-lines were exposed for 2, 5, and 10 minutes.

Dark reaction time was $18 \mathrm{hrs}$

Solvent development with dichloromethane: hexane $(2: 8 \mathrm{v} / \mathrm{v})$.

\section{Comments}

The solvent removed the unexposed material leaving a pattern of lines corresponding to all three lines (complete lines for 2, 5 and 10 minutes). 


\section{Example: OV-225 101:1}

The following example shows the photopatterning of a polysiloxane that contains cyanosubstituents. These increase the dipolarity and basicity of a sensing polymer relative to polydimethyl siloxanes. The polymer in this example is 50\% cyanopropylmethyl substituted and $50 \%$ phenylmethyl substituted, and it contains $1 \%$ vinyl substituents randomly distributed along the chain. The crosslinker is a simple tetrafunctional crosslinker, meaning it has four silicon hydride groups. Thus this example combined a polymer containing very polar functionalities and randomly distributed vinyl groups with a simple molecular silicon-hydride containing crosslinker.

The following materials were used and combined in the proportions indicated.

Cyano-Substituted Polysiloxane

OV-225 (cyanopropylmethylphenylsiloxane, 1 mole\% random vinyl, Mw, 8000)

Si-H Cross-linker 1,3-diphenyl-1,1,3,3-tetrakis(dimethylsiloxy)disiloxane, Mw, 527

\section{Catalyst}

$\operatorname{Pt}(\mathrm{acac})_{2}$

\section{Composition Amounts}

$0.30 \mathrm{~g} \mathrm{OV}-225$ (equates to $2.2 \times 10^{-5}$ equivalents of vinyl)

$0.0029 \mathrm{~g}\left(5.6 \times 10^{-6} \mathrm{~mole}\right)$ diphenyltetrakis(dimethylsiloxy)disiloxane

2 drops of $0.5 \mathrm{M} \mathrm{Pt}(\mathrm{acac})_{2}$ in dichloromethane

$2.3 \mathrm{~mL}$ dichloromethane added

Mass ratio of polymer: cross-linker, 101:1

The silicon wafer was cleaned with ozone-uv. A film was coated onto the wafer surface by spincoating at $3000 \mathrm{rpm}$ using the solution composition described above.

\section{Surface Preparation}

Silicon wafer

Ozone-uv treated for 2 minutes.

\section{Spin Cast Conditions}

$3000 \mathrm{rpm}$

\section{Photopatterning Conditions}

The wafer was exposed to ultraviolet light using the mask aligner system.

Individual arc-lines were exposed for 2,5 , and 10 minutes.

Dark reaction time was $18 \mathrm{hrs}$

Solvent development with dichloromethane: hexane $(2: 8 \mathrm{v} / \mathrm{v})$.

\section{Comments}

The solvent removed the unexposed material leaving a pattern of lines corresponding to all three lines (complete lines for 2, 5 and 10 minutes). 


\section{Example: OV-225 50:1}

The following example shows the photopatterning of a polysiloxane that contains cyanosubstituents. These increase the dipolarity and basicity of a sensing polymer relative to polydimethyl siloxanes. The polymer in this example is 50\% cyanopropylmethyl substituted and $50 \%$ phenylmethyl substituted, and it contains $1 \%$ vinyl substituents randomly distributed along the chain. The crosslinker is a simple tetrafunctional crosslinker, meaning it has four silicon hydride groups. Thus this example combined a polymer containing very polar functionalities and randomly distributed vinyl groups with a simple molecular silicon-hydride containing crosslinker.

The following materials were used and combined in the proportions indicated.

Cyano-Substituted Polysiloxane

OV-225 (cyanopropylmethylphenylsiloxane, 1 mole\% random vinyl, Mw, 8000)

Si-H Cross-linker 1,3-diphenyl-1,1,3,3-tetrakis(dimethylsiloxy)disiloxane, Mw, 527

\section{Catalyst}

$\operatorname{Pt}(\mathrm{acac})_{2}$

\section{Composition Amounts}

$0.24 \mathrm{~g} \mathrm{OV}-225$ (equates to $1.8 \times 10^{-5}$ equivalents of vinyl)

$0.0048 \mathrm{~g}\left(9.1 \times 10^{-6}\right.$ mole) diphenyltetrakis(dimethylsiloxy)disiloxane

2 drops of $0.5 \mathrm{M} \mathrm{Pt}(\mathrm{acac})_{2}$ in dichloromethane

$1.8 \mathrm{~mL}$ dichloromethane added

Mass ratio of polymer: cross-linker, 50:1

The silicon wafer was cleaned with ozone-uv. A film was coated onto the wafer surface by spincoating at $3000 \mathrm{rpm}$ using the solution composition described above.

\section{Surface Preparation}

Silicon wafer

Ozone-uv treated for 2 minutes.

\section{Spin Cast Conditions}

$3000 \mathrm{rpm}$

\section{Photopatterning Conditions}

The wafer was exposed to ultraviolet light using the mask aligner system.

Individual arc-lines were exposed for 2, 5, and 10 minutes.

Dark reaction time was 18 hrs

Solvent development with dichloromethane: hexane $(2: 8 \mathrm{v} / \mathrm{v})$.

\section{Comments}

The solvent removed the unexposed material leaving a pattern of lines corresponding to all three lines (complete lines for 2, 5 and 10 minutes). 


\section{Example: BSP3}

The following example demonstrates the creation of a photopatterned film of a bisphenolcontaining polycarbosilxane polymer. Bisphenol groups make the polymer hydrogen bond acidic, which enhances interactions with basic analytes. Photoinitiation was used to polymerize, crosslink, and graft the polymer. The initial composition consisted of monomer with two allyl groups, an oligomer terminated in silicon hydride groups, a molecular crosslinker and photoactivatable catalyst. The oligomer was synthesized here. Details of the synthesis are presented in the forward section.

The following materials were used and combined in the proportions indicated.

Bisphenol-A Containing Monomer with Allyl Groups

2,2-bis(3-allyl-4-hydroxyphenyl)hexafluoropropane, Mw, 416.4

Si-H Terminated Component

2,2-bis(3-allyl-4-hydroxyphenyl)hexafluoropropane terminated with hexamethyltrisiloxane (BSP

Triad), Mw, 833 if truly a triad, however analysis indicates that on average, it has 5 monomers per oligomer

Multifunctional Molecular Cross-linker

1,3-diphenyl-1,1,3,3-tetrakis(dimethylsiloxy)disiloxane, Mw, 527

\section{Catalyst}

$\operatorname{Pt}(\text { acac })_{2}$

\section{Composition Amounts}

$0.021 \mathrm{~g}\left(5.2 \times 10^{-5}\right.$ mole) 2,2-bis(3-allyl-4-hydroxyphenyl)hexafluoropropane, DN-2/134A, cut 3

$0.086 \mathrm{~g}\left(1.0 \times 10^{-4}\right.$ mole assuming a molecular weight of 833) BSP Triad, DN-2/105, cut 3-4

$0.00054 \mathrm{~g}\left(1.0 \times 10^{-6} \mathrm{~mole}\right)$ diphenyltetrakis(dimethylsiloxy)disiloxane

2 drops of $0.5 \mathrm{M} \mathrm{Pt}(\mathrm{acac})_{2}$ in dichloromethane

$0.81 \mathrm{~mL}$ dichloromethane as solvent for $10 \mathrm{wt} \%$ solution

Mole ratio of triad, monomer, cross-linker, 2:1:0.01 (assuming MW of 833 for triad. Actual mole ratio would be less than $2: 1$.

The silicon wafer was cleaned with ozone-uv and treated with triethoxyvinylsilane. A film was coated onto the wafer surface by spin-coating at $3000 \mathrm{rpm}$ using the solution composition described above. Solution did not wet silicon surface, but ran to low edge. Complete coverage achieved regardless (mottled surface)

\section{Surface Preparation}

Silicon wafer

Ozone-uv treated for 2 minutes, followed by exposure to triethoxyvinylsilane (acetone, refluxing acetone, $24 \mathrm{hrs}$ ).

\section{Spin Cast Conditions \\ $3000 \mathrm{rpm}$}

\section{Photopatterning Conditions}

The wafer was exposed to ultraviolet light using the mask aligner system. 
Individual arc-lines were exposed for 2, 5, and 10 minutes.

Dark reaction time was $18 \mathrm{hrs}$

Solvent development with dichloromethane: hexane $(2: 8 \mathrm{v} / \mathrm{v})$, followed by dichloromethane.

\section{Comments}

The solvent removed the unexposed material leaving a pattern of 3 lines for each time period. Some incomplete sections were apparent in each line. Note that 2 min line was as good as those with extended exposure. 


\section{Distribution}

No. of

Copies

OFFSITE

2 U.S. Department of Energy 1000 Independence Avenue SW Washington D.C. 20585

Attn: M. O'Connell

L. Pitts
No. of

Copies

\section{ONSITE}

17 Pacific Northwest National Laboratory

G. B. Dudder (5)

R. Clemmer (5)

J. W. Grate (5)

K8-93

Technical Report Files (2)

Distr. 1 